\title{
A THEOREM ON DIMENSION
}

\section{L. CURTIS AND G. S. YOUNG}

Every $n$-dimensional separable metric space can be homeomorphically imbedded in the closed $(2 n+1)$-cube $I^{2 n+1}$. This, of course, does not characterize $n$-dimensionality, for spaces of dimension greater than $n$ can be imbedded homeomorphically in $I^{2 n+1}$. A theorem due to Hurewicz ${ }^{1}$ suggests that the existence of a more general kind of mapping into the $n$-cube $I^{n}$ may characterize $n$-dimensionality for compact spaces. For compact spaces this theorem reduces to: If $X, Y$ are compact and separable metric, $f: X \rightarrow Y$ is continuous, and $\operatorname{dim} X \geqq \operatorname{dim} Y$, then there exists $y \in Y$ such that $\operatorname{dim} f^{-1}(y) \geqq \operatorname{dim} X-\operatorname{dim} Y$. This suggests the possibility that if $\operatorname{dim} X$ $=\operatorname{dim} Y$, one may be able to modify the map $f$ slightly to obtain a map $g$, so that each $g^{-1}(y)$ has dimension zero, or is light. While this is not true in general, we show that it is possible if $\operatorname{dim} X=n$ and $Y=I^{n}$.

Lemma 1. Let $P^{n}$ be an $n$-polyhedron and $I^{n}$ be the $n$-cube, both with given simplicial decompositions. If $\psi: P^{n} \rightarrow I^{n}$ is simplicial and $\delta>0$, then there exists a map $\phi: P^{n} \rightarrow I^{n}$ such that:

(1) For any $x \in P^{n},\|\phi(x)-\psi(x)\|<\delta$.

(2) For any $y \in I^{n}, \phi^{-1}(y)$ has at most one point in each (open) simplex of $P^{n}$.

Proof. Let $0<\eta<\min (\delta / 2, \zeta / 2)$ where $\zeta$ is the minimum diameter of the collection of $n$-simplexes of $I^{n}$. For each vertex $q$ in the decomposition of $I^{n}$ let $S^{n-1}(q)=\left\{y \mid y \in I^{n},\|y-q\|=\eta\right\}$. On each $S^{n-1}(q)$ select $\aleph_{0}$ distinct points such that if $q$ and $q^{\prime}$ are two vertices with $q^{\prime} \in$ the closure of the set $\operatorname{St}(q)$, then no $(n-1)$-hyperplane determined by $n$ of the selected points of $S^{n-1}(q)$ coincides with an $(n-1)$-hyperplane determined by $n$ of the selected points of $S^{n-1}\left(q^{\prime}\right)$.

Let $\left\{p_{i}\right\}$ denote the collection of vertices of $P^{n}$. If $\psi^{-1}(q)=U_{p_{i}}$, then let $\left\{\phi^{\prime}\left(p_{i_{j}}\right)\right\}$ be distinct points from the collection defined on $S^{n-1}(q)$. This defines $\phi^{\prime}$ on all the vertices of $P^{n}$, and, if $\sigma_{l}$ is an $l$ simplex, then $\phi^{\prime}$ sends its vertices into $l+1$ independent points of $I^{n}$. Extend $\phi^{\prime}$ linearly on each simplex of $P^{n}$ to obtain a map $\phi: P^{n} \rightarrow I^{n}$. It can be seen that $\phi$ has the required properties.

Definition. A map $f: X \rightarrow Y$ is said to be $\epsilon$-light provided that: For any $y \in Y$, each component of $f^{-1}(y)$ has dia $<\epsilon$.

Presented to the Society, April 28, 1951; received by the editors February 27, 1951.

1 Dimension theory, Hurewicz and Wallman, Princeton, 1948, Theorem VI, 7, p. 91. 
Given two spaces $X, Y$ and a sequence $\left\{\epsilon_{i}\right\} \rightarrow 0$. Let $F_{\epsilon_{i}}$ denote the set of all $\epsilon_{i}$-light maps of $X$ into $Y$. Then $\bigcap_{i} F_{\epsilon_{i}}=$ all light maps $X \rightarrow Y$.

Theorem. Let $X$ be a compact metric space. Then $\operatorname{dim} X \leqq n$ if and only if there exists a light map of $X$ into $I^{n}$.

Proof. Necessity. Let $F$ denote the function space of maps $f: X \rightarrow I^{n}$ with the uniform topology, and denote by $F_{\epsilon}$ the set of $\epsilon$-light elements of $F$.

$(\alpha)$ For any $\epsilon>0, F_{\epsilon}$ is open in $F$.

Suppose that $f \in F_{e}$ and $\left\{h_{i}\right\} \rightarrow f$ with each $h_{i} \in F-F_{\text {c. }}$ Then for each $i$ there is a point $y_{i} \in I^{n}$ and a component $c_{i}$ of $h_{i}^{-1}\left(y_{i}\right)$ such that dia $c_{i} \geqq \epsilon$. Let $y$ be a limit point of $\left\{y_{i}\right\}$. Then $\left\{c_{i}\right\}$ contains a subsequence converging to a closed connected set $C$ with dia $c \geqq \epsilon$. Furthermore $C \subset f^{-1}(y)$, contradicting the assumption that $f \in F_{\epsilon}$.

$(\beta)$ For any $\epsilon>0, F_{\text {e }}$ is dense in $F$.

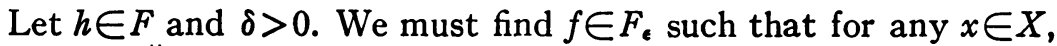
$\|f(x)-h(x)\|<\delta$.

Take a simplicial decomposition of $I^{n}$ into simplexes of dia $<\delta / 2$. If $\left\{q_{i}\right\}$ are the vertices, then $\left\{h^{-1}\left(\operatorname{St}\left(q_{i}\right)\right)\right\}$ is a covering of $X$ by open sets. Take a covering $V$ of $X$ such that

(1) $V$ is a refinement of $\left\{h^{-1}\left(\operatorname{St}\left(q_{i}\right)\right)\right\}$,

(2) Each $v \in V$ has dia $<\epsilon$,

(3) Order $V \leqq n+1$.

Denote by $N(V)$ the nerve of $V$ and by $\zeta$ a barycentric $V$-mapping ${ }^{2}$ $\zeta: X \rightarrow N(V)$. Let $\left\{p_{j}\right\}$ be the collection of vertices of $N(V)$. Then the rule: $\psi^{\prime}\left(p_{j}\right)=$ some $q_{i}$ such that $h\left(v_{j}\right) \subset$ St $\left(q_{i}\right)$ defines a simplicial map in $\psi^{\prime}: N(V) \rightarrow I^{n}$. By the lemma we can find a map $\psi: N(V) \rightarrow I^{n}$ satisfying conditions (1) (with $\delta / 2$ ) and (2). The combined map $f=\psi \zeta$ has the property that $\|f(x)-h(x)\|<\delta$ for every $x \in X$; furthermore $f \in F_{\text {c. }}$ For let $C$ be a connected set in $X$ such that $f(C)$ is a single point $y \in I^{n}$. Then $\zeta(C)$ is a connected set in $N(V)$, but it must be contained $\psi^{-1}(y)$ which is totally disconnected. Thus $\zeta(C)$ is a single point, and, since $\zeta$ is a barycentric $V$-mapping, this implies dia $C<\epsilon$.

$(\gamma)$ The function space $F$ is complete so that we may apply a theorem of Baire stating that the intersection of a countable number of open dense sets of a complete space has an intersection which is dense in the space. Thus if $\left\{\epsilon_{i}\right\} \rightarrow 0$, then the collection of light maps of $X$ into $I^{n}\left(=\bigcap_{i} F_{e_{i}}\right)$ is dense in $F$. This completes the proof of the necessity.

Sufficiency. This follows from the theorem of Hurewicz mentioned in the introduction.

2 Op. cit. Definition V, 9, p. 69. 
Remark 1 . The condition in the theorem that $X$ be compact is important. For if $X$ is the set of points in the plane which have at least one coordinate a rational number, then $\operatorname{dim} X=1$, but there is no light closed map of $X$ into the real line, and, a fortiori, none into the unit interval.

REMARK 2. It is clear that $I^{n}$ could be replaced in the theorem by any $n$-manifold. This contrasts with the imbedding theorem, where there are $n$-dimensional sets that can be imbedded in manifolds of dimension less than $2 n+1$, but cannot be imbedded in $I^{2 n}$.

Added in proof. Our attention has been called to a paper by M. Katětov, On rings of continuous functions, Casopis pro Pěstování Matematiky a Fysiky vol. 75 (1950) pp. 1-16, Mathematical Reviews vol. 12 (1951) p. 119, which apparently contains several theorems closely related to our result, though not the same.

NORTHWESTERN UNIVERSITY AND

UNIVERSITY OF MICHIGAN 